\title{
Vertical transmission of methicillin-resistant Staphylococcus aureus at delivery and its clinical impact: an observational, prospective cohort study
}

\author{
Jumpei Ogura ${ }^{1}$, Yoshihide Inayama ${ }^{2}$, Naoko Sasamoto ${ }^{3}$, Takahiro Hirayama ${ }^{2}$, Tsutomu

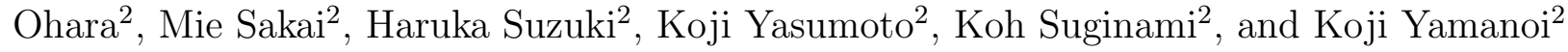 \\ ${ }^{1}$ Kyoto University Graduate School of Medicine Faculty of Medicine \\ ${ }^{2}$ Toyooka Public Hospital Association \\ ${ }^{3}$ Brigham and Women's Hospital and Harvard Medical School
}

February 22, 2021

\begin{abstract}
Objectives: To accurately assess the vertical transmission rate of Methicillin-resistant Staphylococcus aureus (MRSA) and its clinical impacts on pregnant mothers and neonates. Design: A prospective observational cohort study. Setting: Japan. Population: 898 pregnant women and their 905 neonates delivered in gestation week $>32+0$ at Toyooka Hospital from August 2016 to December 2017. Methods: MRSA was cultured from the nasal and vaginal samples taken from mothers at enrollment and the nasal and umbilical samples taken from neonates immediately after their birth. We examined the vertical transmission rate of MRSA in mother-neonate pairs. We used multivariable logistic regression to identify maternal/neonatal adverse outcomes associated with maternal MRSA colonization. Main outcome measures: The frequency of maternal/neonatal MRSA colonization and adverse outcomes. Results: The prevalence of maternal MRSA colonization was 6.1\% (55/898), and that of neonates was $0.8 \%$ (8/905). The prevalence of neonatal MRSA was $12.7 \%$ (7/55 mother-neonate pairs) in the MRSA-positive mothers, whereas it was only $0.12 \%$ (1/843 pairs) in the MRSA-negative mothers (OR: $121,95 \%$ CI: 14.6-1000). When the maternal vaginal-MRSA was positive, the vertical transmission rate was $44.4 \%$ (4/9). Skin and soft tissue infections (SSTIs) developed significantly more frequently in neonates born to the MRSA-positive mothers (OR: 7.47, 95\% CI: 2.50-22.3). Conclusions: The prevalence of MRSA in pregnant women was approximately $6 \%$. The vertical transmission rate of vaginal MRSA was as high as 44.4\%. Maternal MRSA colonization is associated with increased development of neonatal SSTIs via vertical transmission.
\end{abstract}

\section{Title:}

Vertical transmission of methicillin-resistant Staphylococcus aureus at delivery and its clinical impact: an observational, prospective cohort study

\section{Name and affiliation of all authors:}

Jumpei Ogura, Department of Obstetrics and Gynecology, Toyooka public hospital.

1097 Tobera, Toyooka city, Hyogo, Japan. 668-8501

Yoshihide Inayama, Department of Obstetrics and Gynecology, Toyooka public hospital.

1097 Tobera, Toyooka city, Hyogo, Japan. 668-8501

Naoko Sasamoto, Department of Obstetrics and Gynecology, Brigham and Women's Hospital and Harvard Medical School. 
25 Shattuck Street, Boston, MA 02115, USA.

Takahiro Hirayama, Department of Obstetrics and Gynecology, Toyooka public hospital.

1097 Tobera, Toyooka city, Hyogo, Japan. 668-8501

Tsutomu Ohara, Department of Obstetrics and Gynecology, Toyooka public hospital.

1097 Tobera, Toyooka city, Hyogo, Japan. 668-8501

Mie Sakai, Department of Obstetrics and Gynecology, Toyooka public hospital.

1097 Tobera, Toyooka city, Hyogo, Japan. 668-8501

Haruka Suzuki, Department of Obstetrics and Gynecology, Toyooka public hospital.

1097 Tobera, Toyooka city, Hyogo, Japan. 668-8501

Koji Yasumoto, Department of Obstetrics and Gynecology, Toyooka public hospital.

1097 Tobera, Toyooka city, Hyogo, Japan. 668-8501

Koh Suginami, Department of Obstetrics and Gynecology, Toyooka public hospital.

1097 Tobera, Toyooka city, Hyogo, Japan. 668-8501

Koji Yamanoi, Department of Obstetrics and Gynecology, Toyooka public hospital.

1097 Tobera, Toyooka city, Hyogo, Japan. 668-8501

Name and Contact information:

Koji Yamanoi, M.D., Ph.D.,

Department of Obstetrics and Gynecology, Toyooka public hospital.

1094 Tobera, Toyooka city, Hyogo, Japan. 668-8501

Tel: 81-796-22-6111 E-mail:kojiymni@kuhp.kyoto-u.ac.jp

Shortened running title: Vertical transmission of MRSA at delivery.

Abstract:

Objectives: To accurately assess the vertical transmission rate of Methicillin-resistant Staphylococcus aureus (MRSA) and its clinical impacts on pregnant mothers and neonates.

Design: A prospective observational cohort study.

Setting: Japan.

Population: 898 pregnant women and their 905 neonates delivered in gestation week $>32+0$ at Toyooka Hospital from August 2016 to December 2017.

\section{Methods:}

MRSA was cultured from the nasal and vaginal samples taken from mothers at enrollment and the nasal and umbilical samples taken from neonates immediately after their birth. We examined the vertical transmission rate of MRSA in mother-neonate pairs. We used multivariable logistic regression to identify maternal/neonatal adverse outcomes associated with maternal MRSA colonization.

Main outcome measures: The frequency of maternal/neonatal MRSA colonization and adverse outcomes.

\section{Results:}


The prevalence of maternal MRSA colonization was 6.1\% (55/898), and that of neonates was $0.8 \%$ (8/905). The prevalence of neonatal MRSA was $12.7 \%$ (7/55 mother-neonate pairs) in the MRSA-positive mothers, whereas it was only $0.12 \%$ (1/843 pairs) in the MRSA-negative mothers (OR: 121, 95\% CI: 14.6-1000). When the maternal vaginal-MRSA was positive, the vertical transmission rate was $44.4 \%$ (4/9). Skin and soft tissue infections (SSTIs) developed significantly more frequently in neonates born to the MRSA-positive mothers (OR: 7.47, 95\% CI: 2.50-22.3).

\section{Conclusions:}

The prevalence of MRSA in pregnant women was approximately $6 \%$. The vertical transmission rate of vaginal MRSA was as high as $44.4 \%$. Maternal MRSA colonization is associated with increased development of neonatal SSTIs via vertical transmission.

Funding: Toyooka Public Hospital Clinical Research Fund.

Keywords: MRSA, pregnancy, vertical transmission, neonatal infection, SSTIs.

Tweetable abstract:

Approximately $40 \%$ of vaginal MRSA are vertically transmitted at delivery and may increase SSTIs in neonates.

\section{Main text:}

\section{Introduction}

Methicillin-resistant Staphylococcus aureus (MRSA) infections are exceedingly difficult to treat and sometimes result in serious consequences, especially when they occur in a compromised host. Pregnant women are compromised hosts, and MRSA infection is a cause of severe diseases in pregnant women ${ }^{1,2}$. The most important compromised hosts in perinatal care are neonates, especially premature babies, who require admission to the neonatal intensive care unit (NICU). When these populations become infected with MRSA, they easily develop serious complications ${ }^{3-5}$. Therefore, MRSA infection of pregnant women and neonates has significant clinical impact in the obstetric field ${ }^{6}$.

MRSA infection is difficult to treat after it develops. Therefore, we placed great importance on the prevention of MRSA outbreaks. Healthcare providers have the most important role in preventing MRSA, especially in the NICU ${ }^{7}$. Although the role that health providers play remains important, there is no apparent association between the amount of effort put towards preventing MRSA transmission from healthcare providers to neonates and the actual prevention rates of MRSA outbreaks in the NICU ${ }^{8}$. The prevention of MRSA transmission from new neonates admitted to the NICU is also important. The screening of neonates admitted to the NICU for MRSA carriage by the collection of cultures from various sites is common in Japan. However, it takes several days to obtain the culture results. If neonates newly admitted to the NICU are MRSA carriers, MRSA may spread through the NICU during this waiting period. Therefore, it is extremely useful to know information related to MRSA carriage before admission to the NICU. Unfortunately, this information is not available.

MRSA colonization in mothers is sometimes found accidentally, but there is no clinical protocol for management. MRSA is an indigenous bacteria of the skin, and it may be vertically transmitted, like GBS. Therefore, information of maternal MRSA colonization may be useful to estimate the MRSA status of their neonates. However, there is no consensus on the exact frequency of vertical transmission of MRSA at a full term delivery on a large scale.

We performed a Pr ospe ctive cohort study for $v$ ertical tran smission of MRSA $c$ onducte $\mathrm{d}$ in the TAJIM A ar ea (Prevalence-in-TAJIMR study) in Japan to investigate the prevalence of MRSA and its clinical impacts on pregnant women and their full-term neonates.

\section{Methods}


Institutional review board and patient consent.

The PREVALANCE-in-TAJIMR study was approved by the Institutional Review Board of Toyooka Public Hospital (Permission number:109). Written consent was obtained from each pregnant woman for her and her neonate's participation in the study.

Study design, study period and study participants

The Prevalance-in-TAJIMR study is an observational, prospective cohort study in the TAJIMA area, which is the northern area of the Hyogo Prefecture in Japan. Pregnant women at 32 to 36 weeks gestation who visited the Perinatal Medical Center in Toyooka Public Hospital were recruited from August 2016 to December 2017. Women who were $>18$ years of age, willing to comply with the study-related procedures (including nasal swabs, enrollment of her child at birth), and capable of providing written informed consent were enrolled. At the time of enrollment, moistened nasal and vaginal swabs were collected from the mother. Mothers and their neonates were followed until day seven after birth and were assessed of adverse outcomes. MRSA-positive women and their neonates were separately monitored during hospitalization.

Although MRSA can be detected in various sites, they are commonly detected in the nares ${ }^{9,10}$. In addition, we were interested in how often MRSA can be detected in the vagina. Therefore, we screened for maternal MRSA colonization using nasal and vaginal samples in our study. For neonates, we used nasal and umbilical samples for screening, as these sites had the highest detection efficiency according to the previous reports ${ }^{9,10}$.

Data collection at enrollment

The clinical data were collected from our medical records and through in-person interview questionnaires. We collected factors related to maternal background, including maternal age at enrollment, body mass index (BMI), parity, occupation (healthcare provider or not), history of spontaneous abortion, history of infertility treatment, diagnosis of preterm labor at enrollment, usage of antibiotics during current pregnancy, twin pregnancy, diagnosis of gestational diabetes mellitus (GDM) before enrollment, diagnosis of hypertensive disorders of pregnancy (HDP) before enrollment, regular hospital visits outside of regular prenatal examinations, alcohol consumption, smoking history, surgical history, hospitalization during pregnancy, and colonization of GBS in the rectovaginal region.

\section{Study outcomes}

The primary outcome of this study was to determine the prevalence of neonatal MRSA colonization at birth. In addition, we investigated the cumulative incidence of both maternal and neonatal events that occurred in the perinatal period as the secondary outcome. We investigated the following maternal events: preterm delivery before 37 weeks of gestation, cesarean section $(\mathrm{C} / \mathrm{S})$, premature rupture of the membranes $(\mathrm{PROM})$, prolonged PROM duration (over 24 hours), and vacuum or forceps delivery. We investigated the following neonatal events: $\mathrm{pH}$ of the umbilical cord arterial blood, Apgar score (1 minute and 5 minute), fetal weight, admission into the NICU, and development of SSTIs, which may be less serious but are potentially associated with MRSA.

\section{Sample collection and MRSA detection}

All samples were collected using a standardized sterile procedure by midwives and doctors at enrollment and during delivery. In our hospital, the midwives or doctors involved in labor and delivery always applied sterile techniques when touching pregnant women and fetuses, so the specimens collected immediately after delivery are completely unaffected by the delivery personnel. Therefore, if MRSA is detected in the cultures of the fetal samples collected immediately after delivery, it should be considered that the infection is almost entirely due to vertical transmission.

All samples collected in this study were processed at Toyooka Public Hospital. We used a cotton-tipped seed swab $\gamma 1$ (Eiken Chemical Co., Ltd. Tokyo, Japan). Within 24 hours after collection, the swabs were streaked on MS-CFX MRSA-specific agar plates, which consisted of mannitol salt agar with egg yolk supplemented 
with cefoxitin (Nissui, Tokyo, Japan), and the plates were incubated for 24 to 48 hours in ambient air at $35^{\circ} \mathrm{C}$.

Data analysis

Chi-square test or Fisher's exact test was used to examine the associations in categorical variables and unpaired t-test was used to examine the associations among continuous variables. Multivariable logistic regression model was used to determine the independent predictors of the primary outcome (neonatal MRSA colonization) and secondary outcomes. Statistical significance was considered if $\mathrm{p}<0.05$. Odds ratios (ORs) and $95 \%$ confidence intervals $(95 \%$ CI) were calculated and a variable was considered significantly associated with the outcomes if the CI did not include 1.0. Details about statistics method are described in Supplementary material, Method S1.

\section{Funding}

This study was supported by Toyooka Public Hospital Clinical Research Fund.

\section{Results}

Characteristics of the study population.

The flow chart of the study is shown in Supplementary material, Figure S1. Overall, 1,223 pregnant women visited our hospital from the first trimester of pregnancy to 31 weeks of gestation and were eligible for the study. A total of 898 pregnant women agreed to participate and were enrolled in the study. Their clinical characteristics are shown in supplementary material (Table S1). 55 of all pregnant women (6.1\%) were colonized with MRSA according to nasal or vaginal swabs (Figure S1). 46 pregnant women (5.1\%) had MRSA colonization only from the nasal swab, and nine (1.0\%) had MRSA colonization from the vaginal swab (Figure S1). We categorized the 55 MRSA-positive pregnant women as the M-group, and the remaining 843 uninfected pregnant women as the non-M group. These women were followed prospectively, and there was no loss to follow-up.

All mothers had live births, and there were no perinatal deaths during the study period. These 898 mothers, including seven with twin pregnancies, delivered 905 neonates. One twin pregnancy was included in the M-group, which resulted in 56 neonates in the M-group. Six twin pregnancies were included in the non-M group, which resulted in 849 neonates. We monitored the clinical course of all neonates throughout the study period.

Risk factors for maternal MRSA colonization

We first examined the differences in maternal backgrounds between the M-group and the non-M group (Table 1A). Among the maternal background information, the percentages of multiparous mothers and mothers who worked as healthcare providers were significantly higher in the M-group compared to the non-M group. In the M-group, $67.3 \%(37 / 55)$ were multiparous, and $29.1 \%$ (16/55) were healthcare providers. Only $48.4 \%$ (408/843) were multiparous, and 13.8\% (116/843) were healthcare providers in the non-M group (OR: 2.09, 95\% CI: 1.23 to 3.01 and OR: 2.57, 95\%CI: 1.39 to 4.75 , respectively). The frequency of a parity of two or more was also significantly higher in the M-group compared to the non-M group (15/55: $27.3 \%$ vs. 119/843: $14.1 \%$, OR: $2.28,95 \%$ CI: 1.22 to 4.26 ). Although not significant, the proportion of mothers who used antibiotics during pregnancy tended to be higher in the M-group than the non-M group (5/55: $9.1 \%$ vs. 32/843: 3.8\%, OR: $2.53,95 \%$ CI: 0.95 to 6.79 ).

We performed multivariable logistic regression analysis to determine whether multiparity, occupation (healthcare provider), and history of antibiotic use during pregnancy were independently associated with maternal MRSA colonization. We found that multiparity and occupation (healthcare provider) were independent risk factors for MRSA colonization (OR: 2.35, 95\% CI; 1.25-4.42 and OR: 2.58, 95\% CI; 1.39-4.79, respectively, Table 1B). 
We analyzed the association between maternal background and MRSA colonization in the vagina. We compared the maternal backgrounds of vaginal MRSA-positive patients (VM group, $n=9$ ) and vaginal MRSAnegative patients (non-VM group, $\mathrm{n}=889$ ). The results are shown in Table $1 \mathrm{~A}$. The multiparity rate was significantly higher in the VM group compared to the non-VM group (9/9: 100\% vs. 436/889: 49.0\%, OR: 19.74, 95\%CI: 1.15 to 340.4). The proportion of women with a parity of two or more was also significantly higher in the VM group compared to the non-VM group (6/9: 66.6\% vs. 128/889: 14.4\%, OR: 11.89 , 95\%CI: 2.94 to 48.16). There was no significant difference in the proportion of patients who worked as healthcare providers (VM group; 0/9: 0\% vs. non-VM group; 132/889: 14.8\%, OR: 0.30, 95\%CI: 0.017 to 5.20, Table $1 \mathrm{~A})$.

These analyses showed that being multiparous and a healthcare provider were independent risk factors for maternal MRSA colonization. Multiparity, particularly a parity of two or more, was obviously a significant risk factor for vaginal and maternal MRSA colonization.

Vertical transmission of MRSA to neonates at delivery

Of the 905 neonates delivered in the present study, MRSA was detected in the umbilical stump or nasal cavity at the time of delivery in eight neonates $(0.8 \%)$ (Table $2 \mathrm{~A})$. We assessed the primary outcome of this study, the vertical transmission of MRSA to neonates during delivery and analyzed this outcome in mother-neonate pairs.

There were seven pairs $(12.7 \%)$ in which neonates were MRSA-positive at the time of delivery in the Mgroup $(\mathrm{n}=55)$. There was only one pair $(0.12 \%)$ with a MRSA-positive neonate at the time of delivery in the non-M group $(\mathrm{n}=843)$. Maternal MRSA carriers had a significantly higher risk for having neonatal MRSA colonization at the time of delivery (OR 124; 95\% CI 20.3-1393, Table 2A).

Sub-analysis compared the vertical transmission rate of MRSA in mothers with MRSA in only the nasal cavity, mothers with MRSA in the vagina and non-MRSA mothers (Table 2A). We found that the vertical transmission of MRSA occurred significantly more often in mothers with MRSA in only the nasal cavity than non-MRSA mothers (3/46, 6.38\%, OR 59.2, 95\% CI: 6.0-581). Vertical transmission occurred even more frequently in mothers with MRSA in the vagina than non-MRSA mothers (4/ 9, 44.4\%, OR 678.4, 95\% CI, 64.0-7197). We analyzed the vertical transmission of MRSA based on MRSA colonization in the vagina. The vertical transmission of MRSA in the VM group was also significantly higher than the non-V-M group (V-M group: 4/9, 44.4\%, non-V-M group: 4/896, 0.45\%; OR: 178, 95\% CI 39.9-782, Table 2B).

We used a multivariable regression model to analyze the association between maternal MRSA colonization and neonatal MRSA colonization by adjusting for parity. Maternal MRSA colonization remained significantly associated with neonatal MRSA colonization (Table 2C).

We also performed a stratified analysis to determine whether there were certain factors that increased the frequency of MRSA transmission. However, we did not find any factors that increased the vertical transmission rate of MRSA. None of the neonates born via Caesarean section (CS) had MRSA colonization, which suggests that MRSA is transmitted directly by vaginal delivery (Supplementary material, Table S2).

Maternal MRSA colonization was an independent risk factor for neonatal MRSA colonization at the time of delivery in the present analysis. Even when MRSA was detected only in the maternal nasal cavity, $6.38 \%$ of the neonates had MRSA colonization. If the pregnant women had MRSA colonization in the vagina, the vertical transmission rate of MRSA increased to $44.4 \%$ at the time of delivery.

Association between maternal and neonatal adverse outcomes and MRSA colonization

We assessed the relationship between maternal MRSA colonization and the clinical courses of mothers and their neonates. We did not find any significant difference in maternal adverse outcomes in maternal MRSA colonization (Supplementary material, Table S3). However, the frequency of SSTIs in the neonatal period was significantly higher in the neonates of the M-group than the non-M group (M-group; $3 / 56,5.4 \%$, non-M group: $7 / 849,0.82 \%$, OR 7.47, 95\% CI 2.50-22.3, Table 3). 
We performed the same analysis based on MRSA colonization in the vagina (Table 3A). We found that maternal vaginal MRSA colonization was also significantly associated with the occurrence of SSTIs in the neonatal period (VM group: 2/9, 22.2\%, non-VM group: 8/896, 0.89\%, OR:31.7 95\% CI: 5.68-177). Comparison of the frequency of SSTIs in the neonatal period between the VM group and non-M group revealed a significantly higher frequency in the VM group compared to the non-M group (VM group: 2/9, 22.2\%, non-M group: 8/846, 0.82\%, OR:34.4 95\% CI: 6.04-195, Table 3B). We also examined whether factors other than maternal MRSA colonization were associated with SSTIs, and none of these factors had a significant association with SSTIs (Supplementary material, Table S4).

Therefore, neonatal SSTI was significantly associated with maternal MRSA colonization, especially via the vertical transmission of MRSA.

Table 4 shows the details of all neonatal SSTIs. MRSA was the causative bacterium in all three cases in the $\mathrm{M}$-group but only one of the seven cases in the non-M group $(\mathrm{p}=0.033)$. Two of the three cases in the M-group had symptoms on the day after birth, and only one of the seven cases in the non-M group showed symptoms on the day after birth. We classified all symptoms as invasive (eczema, impetigo and abscess) or noninvasive (rash, inflammation). All three cases in the M-group and one case in the non-M group showed invasive symptoms, and the M-group had a significantly higher frequency of invasive symptoms ( $\mathrm{p}=0.033)$.

Most SSTIs resolved spontaneously within a few days or resolved with simple local disinfection procedures. However, one case of MRSA SSTI in the M-group did not resolve with local treatment and required incision and drainage procedures.

\section{Structured Discussion}

\section{Main Findings}

The actual prevalence of MRSA carriers in pregnant women in the TAJIMA area was $6.1 \%$. The proportion of vaginal MRSA carriers was $1 \%$. Being multiparous and a healthcare provider were independent risk factors for maternal MRSA colonization. MRSA was transmitted to neonates predominantly via a vertical route at vaginal delivery, and its frequency was as high as $44.4 \%$ in cases of MRSA-positive vaginas. Maternal MRSA colonization is a risk factor of SSTIs in their neonates.

\section{Interpretation}

Prevalence of MRSA carriers can vary across time and regions ${ }^{6,11,12}$. Our hospital is the only perinatal medical center within 2,000 km2, and approximately $90 \%$ of deliveries in this area are performed in our facility. Therefore we anticipate that this study almost accurately reflects the proportion of MRSA carriers among pregnant women living in this area. As of 2018, the prevalence rate of community-acquired MRSA can be estimated to be approximately $6 \%$ in Japan.

Among the clinical background characteristics related to MRSA carriers in pregnant women, being multiparous and being a healthcare provider were found to be independent risk factors. Several other studies have also shown that multiparous women and healthcare providers are at greater risk for being MRSA carriers $^{7,13,14}$. It is still unclear why the proportion of MRSA carriers is higher among multiparous women. We assume that these women had been to the hospital more frequently for obstetrical and neonatal issues, which might increase the probability of acquiring MRSA.

We observed that the vertical transmission rate of MRSA from the mother to the neonate during delivery was significantly higher in pregnant women who were MRSA carriers compared to those who were not. While MRSA vertical transmission was observed even among pregnant women with MRSA detected only in the nasal cavity, the vertical transmission rate was much higher in women with MRSA detected in the vagina. Considering that MRSA was transmitted at a high rate when detected in the maternal vagina and that no neonates delivered by $\mathrm{C} / \mathrm{S}$ were MRSA positive, most MRSA transmissions are highly likely to be due to direct vertical transmission by vaginal delivery. 
Consistent with several previous reports, our study found that maternal MRSA carrier status had little influence on maternal and neonatal adverse outcomes ${ }^{15,16}$. Interestingly, however, maternal MRSA carrier status was found to be significantly associated with SSTIs in the neonatal period. Although MRSA infections in preterm neonates are known to be associated with poor prognostic outcomes ${ }^{3}$, the clinical significance of MRSA infection in full-term neonates has not been well studied thus far. We revealed for the first time that the vertical transmission of MRSA was associated with increased occurrence of SSTIs in full-term birth neonates.

Given that MRSA is generally found in the epidermis, we think this is a plausible observation and consistent with other reports ${ }^{7,17}$. MRSA was the causative bacterium in the neonates delivered by the three mothers who were MRSA carriers (Table 4). These three patients showed moderate to severe symptoms such as abscess. Moreover, one patient needed an incision and drainage. On the other hand, all patients with SSTIs who were delivered by non-MRSA carriers showed relatively mild symptoms. There was a tendency for MRSA infections to occur shortly after birth, sometimes on the same day they were born. When an SSTI with moderate to severe symptoms, such as abscess, occurs immediately after birth, we may consider that MRSA is the causative bacterium and that his or her mother is a MRSA carrier.

\section{Strengths and Limitations}

The high rate of vertical transmission found in our study differs from the results of previous studies that reported the vertical transmission of MRSA from mothers to their neonates ${ }^{13,15,18-22}$. In these reports, the vertical transmission rate of MRSA was as high as 10\%, and no study reported as high as $40 \%$ observed in this study. This inconsistent observation may in part be due to small sample size in our study with only nine pregnant women detecting MRSA in the vagina. Another possible reason for the high rate of vertical MRSA transmission in our study may be that since the cultures are taken immediately after delivery, a large amount of maternal vaginal secretions can remain and lead to a high detection rate. However, this should be included within vertical transmission and not be considered as a false-positive result. Additionally, the vertical transmission rate of GBS by delivery is reported to be approximately $40 \%{ }^{23,24}$. Taking those factors into account, the vertical transmission rate of $44.4 \%$ (4/9 pairs) for vaginal MRSA may be reasonable.

Although we performed further stratified analyses to determine other factors that would increase the vertical transmission rate, we did not detect any factors because of the low prevalence rate of vaginal MRSA carriers and the limited sample size. Further studies with larger sample sizes are needed to detect factors that could affect the vertical transmission rate of MRSA.

\section{Conclusions}

The current Prevalence-in-TAJIMAR study was the first to report that approximately $6 \%$ of healthy pregnant women had community-acquired MRSA colonization, and 1\% had vaginal MRSA colonization. MRSA was highly transmitted to neonates by vertical transmission during delivery. In particular, when the mother had vaginal MRSA, the vertical transmission rate was approximately 44\%, which is almost equivalent to that of GBS vertical transmission. MRSA transmission can cause SSTIs in full term neonates. It can be worth checking MRSA colonization in preterm mothers because MRSA infection to preterm neonates can be critical $^{18}$. The results of this study will be even helpful, particularly when MRSA is detected in vaginal cultures taken for any other reason during pregnancy. At present, there is no concrete consensus regarding strategies for when MRSA is detected in the vaginal secretions of pregnant women, but we hope that this study will provide insight for future work on this issue.

\section{Disclosure of Interest:}

The authors report no disclosure of interest.

\section{Contribution to Authorship:}

JO, YI, HT, TO, MS, HS, YK, KS and KY were involved in study design, data collection and interpretation. JO, NS and KY were involved in the data analysis. 
All authors revised the manuscript, approved the final version of the manuscript to be published, and agreed to be accountable for all aspects of the work in ensuring that questions related to the accuracy or integrity of any part of the work are appropriately investigated and resolved.

\section{Details of Ethics Approval:}

This study was approved by the Institutional Review Board of Toyooka Public Hospital (Permission number:109, approval: June 22, 2016).

\section{Source of Funding:}

This study was supported by Toyooka Public Hospital Clinical Research Fund.

\section{Acknowledgments:}

The authors would thank S. Tomotaki M.D., Ph.D. for providing valuable input about the neonatal field.

\section{Supporting Information}

Additional supporting information may be found online in the Supporting Information section at the end of the article.

Method S1. Details of statistic methods.

Figure S1. The flow chart of the study.

Table S1. Demographic and clinical characteristics of patients.

Table S2. Stratified analysis for vertical MRSA transmission.

Table S3. Comparison of maternal adverse outcomes between M-group and non-M group, V-M group and non-V-M group.

Table S4. Comparison of clinical background of mother and neonates between neonatal skin infection positive and negative.

\section{References:}

1. Cosma S, Borella F, Carosso A, et al. Osteomyelitis of the pubic symphysis caused by methicillin-resistant Staphylococcus aureus after vaginal delivery: a case report and literature review. BMC Infect Dis 2019 Nov 8;19(1):952.

2. Maeda N, Hagiya H, Takiuchi T, et al. Persistent methicillin-resistant Staphylococcus aureus bacteremia owing to placental abscess. J Infect Chemother 2018 Dec;24(12):975-9.

3. O'Reilly D, O'Connor C, McCallion N, Drew RJ. A retrospective study (2001-2017) of acute and chronic morbidity and mortality associated with Staphylococcus aureus bacteraemia in a tertiary neonatal intensive care unit. Ir J Med Sci 2019 Nov;188(4):1297-301.

4. Tsai MH, Huang SH, Chen CL, et al. Pathogenic bacterial nasopharyngeal colonization and its impact on respiratory diseases in the first year of life: the PATCH Birth Cohort Study. Pediatr Infect Dis J 2015 Jun;34(6):652-8.

5. Butt IJ, Khan S, Butt S, Bhutta S. Frequency and treatment of methicillin resistant Staphylococcus aureus in obstetric and gynaecological sepsis. J Coll Physicians Surg Pak 2013 Oct;23(10):708-10.

6. Beigi RH. Clinical implications of methicillin-resistant Staphylococcus aureus in pregnancy. Curr Opin Obstet Gynecol 2011 Apr;23(2):82-6.

7. Sheffield JS. Methicillin-resistant Staphylococcus aureus in obstetrics. Am J Perinatol 2013 Feb;30(2):1259 . 
8. Parriott AM, Brown JM, Arah OA. Hospital and provider patient volumes, cesarean section rates, and early postpartum invasive methicillin-resistant Staphylococcus aureus infection. Am J Infect Control 2014 Feb;42(2):156-9.

9. Zamfir M, Adler AC, Kolb S, et al. Evaluation of sampling locations in pregnant women and newborns for the detection of colonisation with antibiotic-resistant bacteria. Eur J Clin Microbiol Infect Dis 2017 Oct;36(10):1819-26.

10. Cursino MA, Garcia CP, Lobo RD, et al. Performance of surveillance cultures at different body sites to identify asymptomatic Staphylococcus aureus carriers. Diagn Microbiol Infect Dis 2012 Dec;74(4):343-8.

11. Andrews WW, Schelonka R, Waites K, Stamm A, Cliver SP, Moser S. Genital tract methicillinresistant Staphylococcus aureus: risk of vertical transmission in pregnant women. Obstet Gynecol 2008 Jan;111(1):113-8.

12. Beigi R, Hanrahan J. Staphylococcus aureus and MRSA colonization rates among gravidas admitted to labor and delivery: a pilot study. Infect Dis Obstet Gynecol 2007;2007:70876.

13. Top KA, Buet A, Whittier S, Ratner AJ, Saiman L. Predictors of Staphylococcus aureus Rectovaginal Colonization in Pregnant Women and Risk for Maternal and Neonatal Infections. J Pediatric Infect Dis Soc 2012 Mar;1(1):7-15.

14. Wang B, Suh KN, Muldoon KA, et al. Risk Factors for Methicillin-Resistant Staphylococcus aureus (MRSA) Colonization Among Patients Admitted to Obstetrical Units: A Nested Case-Control Study. J Obstet Gynaecol Can 2018 Jun;40(6):669-76.

15. Gray JW, Suviste J. Three years' experience of screening for meticillin-resistant Staphylococcus aureus in obstetrics. J Hosp Infect 2013 Jan;83(1):61-3.

16. Johnson NB, Hayes LD, Brown K, et al. CDC National Health Report: leading causes of morbidity and mortality and associated behavioral risk and protective factors-United States, 2005-2013. MMWR Suppl 2014 Oct 31;63(4):3-27.

17. Kriebs JM. Staphylococcus Infections in Pregnancy: Maternal and Neonatal Risks. J Perinat Neonatal Nurs 2016 Apr-Jun;30(2):115-23.

18. Lazenby GB, Soper DE, Beardsley W, Salgado CD. Methicillin-resistant Staphylococcus aureus colonization among women admitted for preterm delivery. Am J Obstet Gynecol 2012 Apr;206(4):329 e1-5.

19. Patel RI, Kaufman HK. Nasopharyngeal carriage of methicillin-resistant Staphylococcus aureus: incidence and outcomes in pregnant women. J Am Osteopath Assoc 2011 Jun;111(6):389-95.

20. Schaumburg F, Alabi AS, Mombo-Ngoma G, et al. Transmission of Staphylococcus aureus between mothers and infants in an African setting. Clin Microbiol Infect 2014 Jun;20(6):O390-6.

21. Reiss-Mandel A, Rubin C, Maayan-Mezger A, et al. Patterns and Predictors of Staphylococcus aureus Carriage during the First Year of Life: a Longitudinal Study. J Clin Microbiol 2019 Sep;57(9).

22. Top KA, Huard RC, Fox Z, et al. Trends in methicillin-resistant Staphylococcus aureus anovaginal colonization in pregnant women in 2005 versus 2009. J Clin Microbiol 2010 Oct;48(10):3675-80.

23. Saha SK, Ahmed ZB, Modak JK, et al. Group B Streptococcus among Pregnant Women and Newborns in Mirzapur, Bangladesh: Colonization, Vertical Transmission, and Serotype Distribution. J Clin Microbiol 2017 Aug;55(8):2406-12.

24. Yadeta TA, Worku A, Egata G, Seyoum B, Marami D, Berhane Y. Vertical transmission of group B Streptococcus and associated factors among pregnant women: a cross-sectional study, Eastern Ethiopia. Infect Drug Resist 2018;11:397-404.

Tables: 
Table 1: Comparison of clinical backgrounds between the M-group and non-M group, V-M group and nonV-M group

Comparison of clinical backgrounds between the M-group and non-M group, VM group and non-VM group. chi-squared analysis was performed.

\begin{tabular}{lllllll}
\hline Parameters & $\mathrm{M}$ & non-M & p-value & VM & non-VM & p-value \\
\hline & -group & -group & & -group & -group & \\
& $(\mathrm{N}=55)$ & $(\mathrm{N}=843)$ & & $(\mathrm{N}=9)$ & $(\mathrm{N}=889)$ & \\
Age, median (interquartile range) & 30 & 31 & 0.3 & 32 & 31 & 0.48 \\
& $(28.0-34.0)$ & $(28.0-34.0)$ & & $(28.5-35.0)$ & $(28.0-34.0)$ & \\
BMI, median (interquartile range) & 21.19 & 20.34 & 0.59 & 20.43 & 20.32 & 0.17 \\
& $(18.6-23.4)$ & $(19.0-22.4)$ & & $(19.8-22.8)$ & $(18.9-22.4)$ & \\
Multipara & $37(67.3)$ & $408(48.4)$ & 0.0078 & $9(100)$ & $436(49.0)$ & 0.0017 \\
Parity>=2, & $15(27.3)$ & $119(14.1)$ & 0.017 & $6(66.7)$ & $128(14.4)$ & 0.0006 \\
Health provider, & $16(29.1)$ & $116(13.8)$ & 0.0048 & $0(0.0)$ & $132(14.8)$ & 0.37 \\
History of SA, & $14(25.5)$ & $244(28.9)$ & 0.65 & $3(33.3)$ & $255(28.7)$ & 0.73 \\
History of ART, & $4(7.3)$ & $63(7.5)$ & 0.79 & $0(0.0)$ & $67(7.54)$ & 1 \\
Preterm labor at enrollment, & $4(7.3)$ & $56(6.6)$ & 0.78 & $0(0.0)$ & $60(6.75)$ & 1 \\
Use of antibiotics during pregnancy & $5(9.1)$ & $32(3.8)$ & 0.069 & $1(11.1)$ & $36(4.05)$ & 0.32 \\
twin pregnancy & $1(1.8)$ & $6(0.72)$ & & $0(0.0)$ & $7(0.79)$ & \\
GDM & $2(3.6)$ & $38(4.5)$ & 1 & $0(0.0)$ & $40(4.50)$ & 1 \\
HDP & $5(9.1)$ & $62(7.4)$ & 0.59 & $0(0.0)$ & $67(7.54)$ & 1 \\
Regular visit & $17(30.9)$ & $188(22.3)$ & 0.45 & $3(33.3)$ & $202(22.7)$ & 0.43 \\
Alcohol & $1(1.8)$ & $18(2.1)$ & 1 & $0(0.0)$ & $19(2.14)$ & 1 \\
Smoking & $1(1.8)$ & $37(4.4)$ & 0.72 & $0(0.0)$ & $38(4.27)$ & 1 \\
History of operation & $3(5.5)$ & $64(7.6)$ & 0.79 & $2(22.2)$ & $65(7.31)$ & 0.14 \\
Hospitalization during pregnancy & $1(1.8)$ & $14(1.7)$ & 0.62 & $0(0.0)$ & $15(1.69)$ & 1 \\
GBS & $10(18.2)$ & $104(12.3)$ & 0.21 & $1(11.1)$ & $113(12.7)$ & 1 \\
\hline
\end{tabular}

B: Odds of maternal carrier of MRSA; multivariant logistic regression analysis

\begin{tabular}{lll}
\hline Parameter & OR (95\%CI) & p-value \\
\hline Multiparous & $2.35(1.25-4.42)$ & 0.0078 \\
Health provider & $2.58(1.39-4.79)$ & 0.0027 \\
History of antibiotics during pregnancy & $2.37(0.87-6.42)$ & 0.1 \\
\hline
\end{tabular}

Note. - M-group, Maternal MRSA-positive group; non-M group, Maternal MRSA- negative group; VMgroup, Maternal Vaginal MRSA positive group, Non-VM- group, Maternal Vaginal MRSA negative group; SA, spontaneous abortion; ART, artificial reproductive technique; GDM, gestational diabetes mellitus; HDP, Hypertensive disorders of pregnancy.

Values are presented as $\mathrm{n}(\%)$ or as otherwise indicated.

Table 2: Vertical transmission of MRSA from mother to neonates

A: Odds of vertical MRSA transmission based on maternal MRSA colonization status

\begin{tabular}{lllll}
\hline Maternal MRSA infection status & $\mathrm{n}$ & Neonatal MRSA positive, $\mathrm{n}(\%)$ & OR $(95 \% \mathrm{CI})$ & $\mathrm{p}$-value \\
\hline non-M group & 849 & $1(0.12)$ & ref &
\end{tabular}




\begin{tabular}{lllll}
\hline Maternal MRSA infection status & $\mathrm{n}$ & Neonatal MRSA positive, $\mathrm{n}(\%)$ & OR $(95 \% \mathrm{CI})$ & $\mathrm{p}$-value \\
\hline M-group & 55 & $7(12.5)$ & $124(14.9-1026)$ & $<0.0001$ \\
nasal-M positive & 46 & $3(6.38)$ & $59.2(6.0-581)$ & 0.0005 \\
V-M group & 9 & $4(44.4)$ & $678(64.0-7197)$ & $<0.0001$ \\
\hline
\end{tabular}

B: Odds of vertical MRSA transmission based on maternal MRSA colonization in the vagina

\begin{tabular}{lllll}
\hline Maternal MRSA infection status & $\mathrm{n}$ & Neonatal MRSA positive, $\mathrm{n}(\%)$ & OR $(95 \% \mathrm{CI})$ & $\mathrm{p}$-value \\
\hline non-V-M group & 896 & $4(0.45)$ & ref & \\
V-M group & 9 & $4(44.4)$ & $178(39.9-782)$ & $<0.0001$ \\
\hline
\end{tabular}

C: Odds of Vertical transmission of MRSA; multivariant logistic regression analysis

\begin{tabular}{lll}
\hline Parameter & OR $(95 \% \mathrm{CI})$ & $\mathrm{p}$-value \\
\hline Maternal MRSA carrier & $158(18.0-1380)$ & $<0.0001$ \\
Multiparous & $0.84(0.14-4.92)$ & 0.85 \\
\hline
\end{tabular}

Note. - M-group, Maternal MRSA positive group; non-M group, Maternal MRSA negative group; nasal-M positive, mother with MRSA in only the nasal cavity; V-M group, Maternal vaginal MRSA-positive group; non-V-M group, Maternal vaginal MRSA-negative group.

Table 3: Comparison of neonatal adverse outcomes between the M-group and non-M group, V-M group and non-V-M group

A: Univariant analysis between the M-group and non-M group, V-M group and non-V-M group. Chi-squared analysis was performed.

\begin{tabular}{|c|c|c|c|c|c|c|c|}
\hline Characterist & $\begin{array}{l}\text { M-group } \\
\text { s(N=56) }\end{array}$ & $\begin{array}{l}\text { non-M } \\
\text { group } \\
(\mathrm{N}=849)\end{array}$ & p-value & $\begin{array}{l}\text { VM-group } \\
(\mathrm{n}=9)\end{array}$ & $\begin{array}{l}\text { VM-group } \\
(\mathrm{n}=9)\end{array}$ & $\begin{array}{l}\text { non-VM } \\
\text { group } \\
(\mathrm{n}=896)\end{array}$ & p-value \\
\hline $\mathrm{pH}<7.15$ & $4(7.14)$ & $35(4.12 \%)$ & 0.295 & & 0 & $39(4.35 \%)$ & 1.0 \\
\hline $\begin{array}{l}\text { AP } 1 \\
\min [?] 4\end{array}$ & $1(1.79)$ & $14(1.65 \%)$ & 1.0 & & 0 & $15(1.67 \%)$ & 1.0 \\
\hline $\begin{array}{l}\text { AP } 5 \\
\min [?] 7\end{array}$ & $0(0.0)$ & $2(0.24 \%)$ & 1.0 & & 0 & $2(0.22 \%)$ & 1.0 \\
\hline $\begin{array}{l}\text { Fetal } \\
\text { weight } \\
<2500\end{array}$ & $5(8.93)$ & $77(9.07 \%)$ & 1.0 & & 0 & $82(9.15 \%)$ & 1.0 \\
\hline $\begin{array}{l}\text { Admission } \\
\text { of NICU }\end{array}$ & $7(12.5)$ & $116(13.66 \%)$ & 1.0 & & 0 & $123(13.73 \%)$ & 0.618 \\
\hline $\begin{array}{l}\text { Neonatal } \\
\text { SSTIs }\end{array}$ & $3(5.4)$ & $7(0.82 \%)$ & 0.0197 & & $2(22.22 \%)$ & $8(0.89 \%)$ & 0.0038 \\
\hline
\end{tabular}

B: Odds of development of neonatal skin infection based on maternal MRSA colonization status. 


\begin{tabular}{|c|c|c|c|c|}
\hline Maternal MRSA colonization status & $\mathrm{n}$ & Neonatal skin infection, $\mathrm{n}(\%)$ & OR $(95 \% \mathrm{CI})$ & p-value \\
\hline non-M group & 849 & $7(0.82)$ & ref & \\
\hline M-group & 56 & $3(5.4)$ & $6.81(1.71-27.1)$ & 0.0197 \\
\hline nasal-M positive & 47 & $1(2.13)$ & $2.61(0.315-21.7)$ & 0.35 \\
\hline V-M group & 9 & $2(22.2)$ & $34.4(6.04-195)$ & 0.0034 \\
\hline
\end{tabular}

Note. - M-group, Maternal MRSA positive group; non-M group, Maternal MRSA negative group; V-M group, Maternal vaginal MRSA-positive group; non-VM group, Maternal vaginal MRSA-negative group; AP, Apgar score; nasal-M positive, mother with MRSA in only the nasal cavity; V-M group, Maternal vaginal MRSA-positive group.

Table4: Maternal and clinical backgrounds and clinical course of neonates who suffered from skin infection.

\begin{tabular}{|c|c|c|c|c|c|c|c|c|c|c|}
\hline Group & Location & $\begin{array}{l}\text { Fetal } \\
\text { MRSA }\end{array}$ & species & location & $\begin{array}{l}\text { Onset } \\
\text { day }\end{array}$ & symptom & outcome & Weeks & Weight & $\mathrm{pH}$ \\
\hline $\mathrm{M}$ & $\mathrm{N}, \mathrm{V}$ & $\begin{array}{l}\mathrm{Na} \\
\mathrm{Um}\end{array}$ & MRSA & Umbilical & day1 & eczema & $\mathrm{SR}$ & 40 & 2600 & 7.185 \\
\hline M & $\mathrm{N}, \mathrm{V}$ & $\begin{array}{l}\mathrm{Na} \\
\mathrm{Um}\end{array}$ & MRSA & $\begin{array}{l}\text { Body } \\
\text { trunk, } \\
\text { left } \\
\text { neck }\end{array}$ & day1 & $\begin{array}{l}\text { impetigo, } \\
\text { abscess }\end{array}$ & Drainage & 39 & 3485 & 7.273 \\
\hline M & $\mathrm{N}$, & None & MRSA & Axilla & day3 & abscess & $\mathrm{LD}$ & 40 & 3235 & 7.139 \\
\hline $\begin{array}{l}\text { non- } \\
\text { M }\end{array}$ & & None & $\begin{array}{l}\text { GBS, } \\
\text { Ecoli }\end{array}$ & Axilla & day1 & Inflamed & $\mathrm{LD}$ & 39 & 3020 & 7.385 \\
\hline $\begin{array}{l}\text { non- } \\
\text { M }\end{array}$ & & None & MSSA & $\begin{array}{l}\text { Neck, } \\
\text { Axilla }\end{array}$ & day2 & Inflamed & $\mathrm{LD}$ & 40 & 3245 & 7.287 \\
\hline $\begin{array}{l}\text { non- } \\
\text { M }\end{array}$ & & None & MRSA & $\begin{array}{l}\text { Head, } \\
\text { Chest, } \\
\text { scrotum }\end{array}$ & day3 & rush & $\mathrm{LD}$ & 40 & 3565 & 7.314 \\
\hline non-M & & None & $\begin{array}{l}\text { Entero. } \\
\text { faecalis }\end{array}$ & jaw & day3 & impetigo & $\mathrm{SR}$ & 40 & 3965 & 7.334 \\
\hline $\begin{array}{l}\text { non- } \\
\text { M }\end{array}$ & & None & E.coli & Umbilical & day4 & Inflamed & $\mathrm{SR}$ & 37 & 2940 & 7.349 \\
\hline $\begin{array}{l}\text { non- } \\
\text { M }\end{array}$ & & None & E.coli & Axilla & day 5 & rush & $\mathrm{LD}$ & 40 & 2875 & 7.251 \\
\hline $\begin{array}{l}\text { non- } \\
\text { M }\end{array}$ & & None & $\begin{array}{l}\text { GBS } \\
\text { E.coli }\end{array}$ & Axilla & day 6 & Inflamed & $\mathrm{LD}$ & 38 & 2910 & 7.204 \\
\hline
\end{tabular}

Note. - M, Maternal MRSA-positive; non-M, Maternal MRSA-negative; Spontaneous remission, SR; Local disinfection, LD; N, Nasal cavity; V, Vagina; Weeks, gestational weeks at delivery; AP: Apgar score. 